\title{
Equitable Post-COVID-19 Care: A Practical Framework to Integrate Health Equity in Diabetes Management
}

Osagie Ebekozien, MD, MPH, Ori Odugbesan, MD, MPH, Nicole Rioles, MA, Shideh Majidi, MD, Nana-Hawa Yayah Jones, MD, and Manmohan Kamboj, MD

H ealth equity has been described as the opportunity for all persons to obtain their highest level of health possible. ${ }^{1}$ Unfortunately, even with advances in technology and care practices, disparities persist in health care outcomes. Disparities in prevalence, prognosis, and outcomes still exist in diabetes management. ${ }^{2}$ Non-Hispanic Black and/or Hispanic populations are more likely to have worse glycemic control, ${ }^{3,4}$ to encounter more barriers in access to care, ${ }^{5}$ and to have higher levels of acute complications, ${ }^{4}$ and to use advanced technologies less frequently. ${ }^{4}$ Diabetes is one of the preexisting conditions that increase morbidity and mortality in COVID-19.6,7 Unfortunately, adverse outcomes from COVID-19 also disproportionately impact a specific vulnerable population. ${ }^{8,9}$ The urgent transition to managing diabetes remotely during the COVID-19 pandemic may exacerbate long-term inequities because some vulnerable patients might not have access to technology devices necessary for effective remote management.

Here, we describe how quality improvement (QI) tools and principles can be adapted into a framework for advancing health equity. Specifically, we describe a 10-step framework that may be applied in diabetes care management to achieve improvement, using a hypothetical example of increasing use of continuous glucose monitors (CGMs) among patients with type 1 diabetes mellitus. ${ }^{10}$ This framework was developed to address the literature gap on practical ways health care providers can address inequities using Ql principles, and was implemented by 1 of the authors at a local public health department. ${ }^{11}$ The framework's iterative and comprehensive design makes it ideal for addressing inequities in chronic diseases like diabetes, which have multiple root causes with no easy solutions. The improvement program pilot received a national model practice award. ${ }^{11,12}$

\section{0-Step Framework}

Step 1: Review program/project baseline data for existing disparities. Diabetes programs and routine Ql processes encourage existing data review to determine how effective the current system is working and if the existing process has a predictable pattern. ${ }^{13,14}$ Our equity-revised framework proposes a more in-depth review to stratify baseline data based on factors that might contribute to inequities, including race, age, income levels, ethnicity, language, sexual orientation, insurance type, and zip code. This process will identify patients not served or unfairly impacted due to socioeconomic factors. For example, using the hypothetical example of improving CGM use, a team completes a preliminary data review and determines that baseline CGM use is 30\% in the clinic population. However, in a review to assess for disparities, they also identify that patients on public insurance have a significantly lower CGM uptake of only $15 \%$.

Step 2: Build an equitable project team, including patients with lived experiences. Routine projects typically have clinicians, administrative staff, and analytic staff as members of their team. In a post-COVID-19 world, every team needs to learn directly from people impacted and share decision-making power. The traditional approach to receiving feedback has generally been to collect responses using surveys or focus groups. We propose that indi-

From T1D Exchange, Boston, MA (Dr. Ebekozien, Dr. Odugbesan, and Nicole Rioles); Barbara Davis Center, University of Colorado, Boulder, CO (Dr. Majidi); Cincinnati Children's Hospital Medical Center, Cincinnati, $\mathrm{OH}$ (Dr. Jones); and Nationwide Children's Hospital, Columbus, $\mathrm{OH}$ (Dr. Kamboj). 


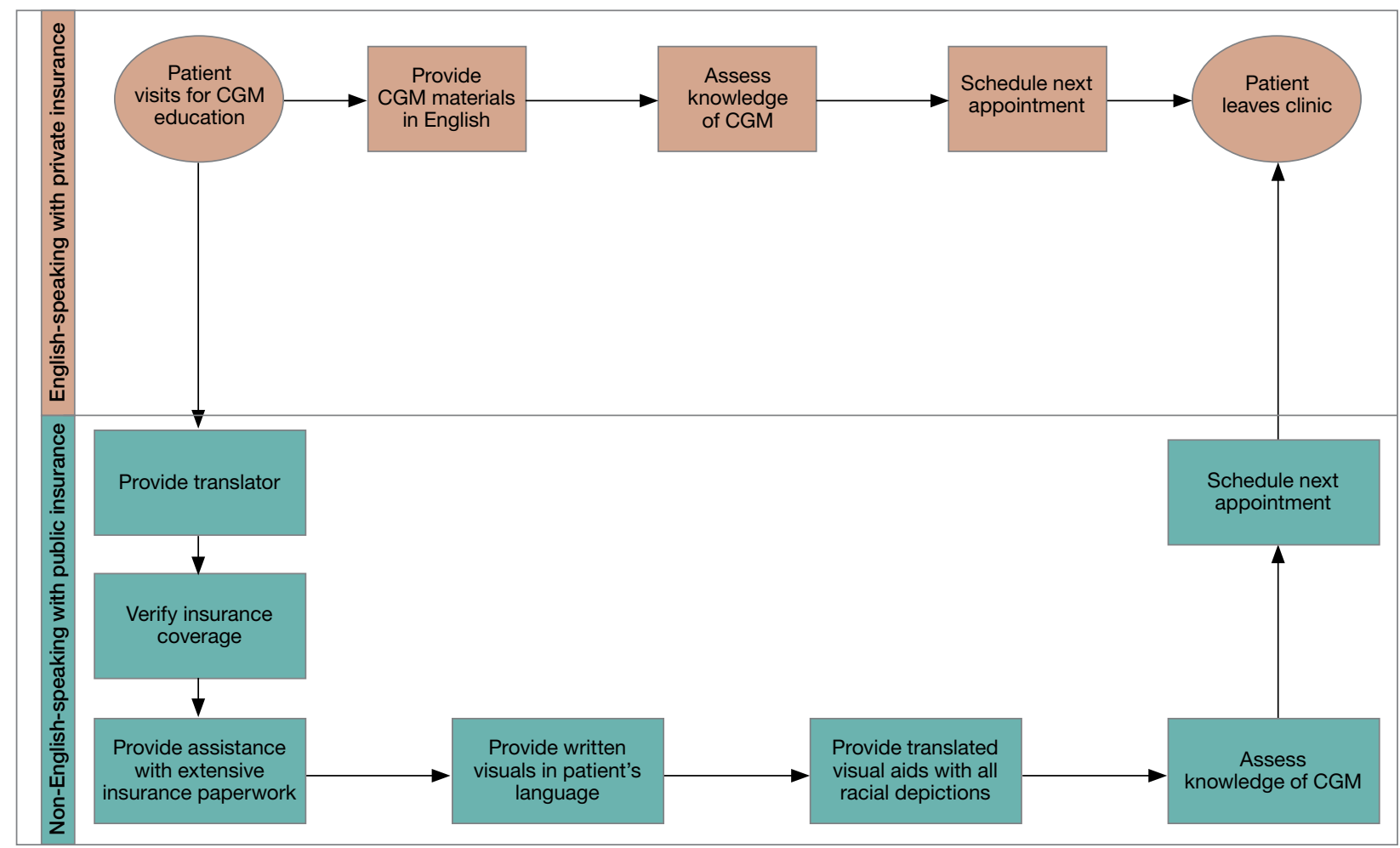

Figure 1. Process map with equity included. The process map illustrates the flow of how patients come into the clinic and are matched with educational materials to support getting them on a continuous glucose monitor (CGM). Workflow highlights the different processes required for patients with public versus private insurances and what resources are appropriate, depending on the patient's preferred language spoken.

viduals/families who are disproportionately impacted be included as active members on QI teams. For example, in the hypothetical example of the CGM project, team members would include patients with type 1 diabetes who are on public insurance and their families.

Step 3: Develop equity-focused goals. The traditional program involves the development of aims that are SMART (specific, measurable, achievable, realistic, time-bound). ${ }^{15}$ The proposed framework encourages the inclusion of equity-revised goals (SMARTer) using insights from Steps 1 and 2. For example, your typical smart goal might be to increase the percentage of patients using CGM by $20 \%$ in 6 months, while a SMARTer goal would be to increase the proportion of patients using CGM by $20 \%$ and reduce the disparities among public and private insurance patients by $30 \%$ in 6 months.

Step 4: Identify inequitable processes/pathways. Traditional QI programs might use a process map or flow diagram to depict the current state of a process visually. ${ }^{16}$ For example, in Figure 1, the process map diagram depicts some differences in the process for patients with public insurance as opposed to those with private insurance. The framework also advocates for using visual tools like process maps to depict how there might be inequitable pathways in a system. Visually identifying inequitable processes/pathways can help a team see barriers, address challenges, and pilot innovative solutions.

Step 5: Identify how socioeconomic factors are contributing to the current outcome. A good understanding of factors that contribute to the problem is an essential part of finding fundamental solutions. The fishbone diagram ${ }^{16}$ is a visualization tool used to identify contributing factors. When investigating contributing factors, it is commonplace to identify factors that fit into 1 of 5 categories: people, process, place, product, and policies (5 Ps). An equity-focused process will include equity as a new major factor category, and the socioeconomic impacts that contribute to inequities will be brainstormed and visually represented. For example, in the hypothetical CGM improvement example, an equity 


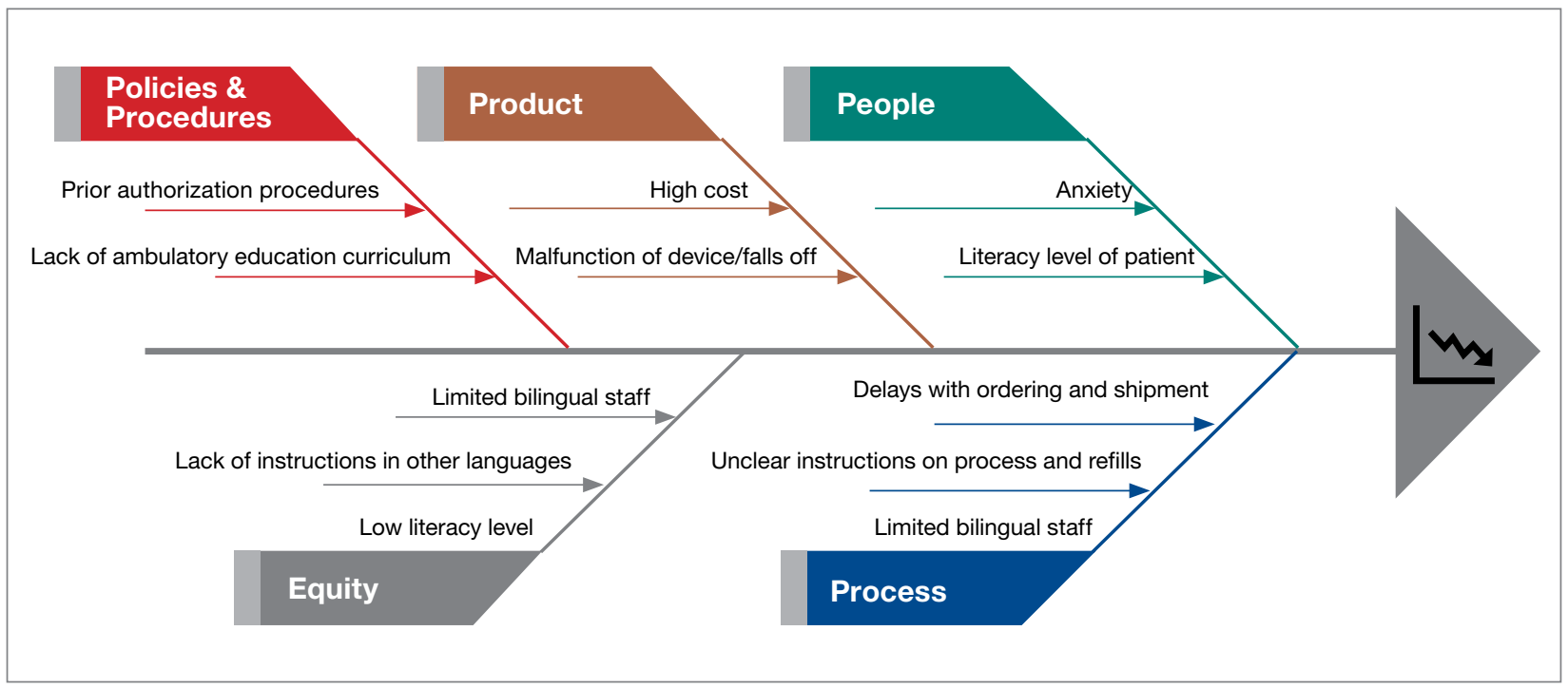

Figure 2. Fishbone diagram with equity included. In this example, the fishbone is divided into 5 categories: policies and procedures, product, people, equity, and process.

contributing factor is extensive CGM application paperwork for patients on public insurance as compared to those on private insurance. Figure 2 shows equity integrated into a fishbone diagram.

Step 6: Brainstorm possible improvements. Potential improvement ideas for the hypothetical CGM example might include redesigning the existing workflow, piloting CGM educational classes, and using a CGM barrier assessment tool to identify and address barriers to adoption.

Step 7: Use the decision matrix with equity as a criterion to prioritize improvement ideas. Decision matrix ${ }^{15}$ is a great tool that is frequently used to help teams prioritize potential ideas. Project team members must decide what criteria are important in prioritizing ideas to implement. Common criteria include implementation cost, time, and resources, but in addition to the common criteria, the team can specify "impact on equity" as one of their criteria, alongside other standard criteria like impact.

Step 8: Test one small change at a time. This step is consistent with other traditional improvement models using the Plan, Do, Study, Act (PDSA) model for improvement. ${ }^{17}$ During this phase, the team should make predictions on the expected impact of the intervention on outcomes. For example, in the hypothetical example, the team predicts that testing and expanding CGM classes will reduce disparities among public versus private health insurance users by $5 \%$ and increase overall CGM uptake by $10 \%$.

Step 9: Measure and compare results with predictions to identify inequitable practices or consequences. After each test of change, the team should review the results, including implementation cost considerations, and compare them to the predictions in the earlier step. The team should also document the potential reasons why their predictions were correct or inaccurate, and whether there were any unforeseen outcomes from the intervention.

Step 10: Celebrate small wins and repeat the process. Making fundamental and equitable changes takes time. This framework aimed at undoing inequities, particularly those inequities that have been amplified by the COVID-19 pandemic, is iterative and ongoing. ${ }^{18,19}$ Not every test of change will impact the outcome or reduce inequity, but over time, each change will impact the next, generating sustainable effects.

\section{Conclusion}

There are ongoing studies examining the adverse outcomes and potential health inequities for patients with diabetes impacted by COVID-19. ${ }^{20}$ Health care providers 
need to plan for post-COVID-19 care, keeping in mind that the pandemic might worsen already existing health disparities in diabetes management. ${ }^{3,4,21}$ This work will involve an intentional approach to address structural and systemic racism. ${ }^{22}$ Therefore, the work of building health equity solutions must be rooted in racial justice, economic equity, and equitable access to health care and education.

Initiatives like this are currently being funded through foundation grants as well as state and federal research or program grants. Regional and national payors, including the Centers for Medicare \& Medicaid Services, are currently piloting long-term sustainable funding models through programs like accountable care organizations and the Accountable Health Communities Model. ${ }^{23}$

Health systems can successfully address health equity and racial justice, using a framework as described above, to identify determinants of health, develop policies to expand access to care for the most vulnerable patients, distribute decision-making power, and train staff by naming structural racism as a driver of health inequities.

Acknowledgment: The authors acknowledge the contributions of patients, families, diabetes care teams, and collaborators within the T1D Exchange Quality Improvement Collaborative, who continually seek to improve care and outcomes for people living with diabetes.

Corresponding author: Osagie Ebekozien, MD, 11 Avenue De La Fayette, Boston, MA 02115; oebekozien@t1dexchange.org.

Financial disclosures: None.

Funding: T1D Exchange QI Collaborative is funded by The Leona M. and Harry B. Helmsley Charitable Trust. No specific funding was received for this manuscript or the development of this framework.

Keywords: type 1 diabetes; quality improvement; QI framework; racial justice; health disparities.

doi:10.12788/jcom.0031

\section{References}

1. American Public Health Association Health Equity web site. https:// www.apha.org/topics-and-issues/health-equity. Accessed June 4, 2020.

2. Lado J, Lipman T. Racial and ethnic disparities in the incidence, treatment, and outcomes of youth with type 1 diabetes. Endocrinol Metab Clin North Am. 2016;45:453-461.

3. Kahkoska AR, Shay CM, Crandell J, et al. Association of race and ethnicity with glycemic control and hemoglobin A1c levels in youth with type 1 diabetes. JAMA Netw Open. 2018;1:e181851.
4. Willi SM, Miller KM, DiMeglio LA, et al; T1D Exchange Clinic Network. Racial-ethnic disparities in management and outcomes among children with type 1 diabetes. Pediatrics. 2015;135:424-434.

5. Valenzuela JM, Seid M, Waitzfelder B, et al. Prevalence of and disparities in barriers to care experienced by youth with type 1 diabetes. J Pediatr. 2014;164:1369-1375.

6. Hussain A, Bhowmik B, do Vale Moreira NC. COVID-19 and diabetes: Knowledge in progress. Diabetes Res Clin Pract. 2020;162:108142.

7. Bhatraju PK, Ghassemieh BJ, Nichols M, et al. Covid-19 in critically ill patients in the Seattle Region - case series. N Engl J Med. 2020;382:2012-2022.

8. Laurencin CT, McClinton A. The COVID-19 pandemic: a call to action to identify and address racial and ethnic disparities. J Racial Ethn Health Disparities. 2020;7:398-402.

9. Shah M, Sachdeva M, Dodiuk-Gad RP. COVID-19 and racial disparities. J Am Acad Dermatol. 2020;83:e35.

10. Ebekozien O, Rioles N, DeSalvo D, et al. Improving continuous glucose monitoring (CGM) use across national centers: results from the T1D Exchange Quality Improvement Collaborative (T1DX-QI). Diabetes. 2020;69(Supplement 1):145-LB.

11. Ebekozien $\mathrm{O}$. Q $\mathrm{Q}$ methodology to address health equity. Presented at American Society of Quality BOSCON 2018; Boston, MA; March 19 and 20, 2018.

12. 2019 Model Practice Award, Building A Culture of Improvement. National Association of County and City Health Officials web site. www.naccho.org/membership/awards/model-practices. Accessed June 4, 2020.

13. Nuckols TK, Keeler E, Anderson LJ, et al. Economic evaluation of quality improvement interventions designed to improve glycemic control in diabetes: a systematic review and weighted regression analysis. Diabetes Care. 2018;41:985-993.

14. Rossi MC, Nicolucci A, Arcangeli A, et al. Baseline quality-of-care data from a quality-improvement program implemented by a network of diabetes outpatient clinics. Diabetes Care. 2008;31:2166-2168.

15. McQuillan RF, Silver SA, Harel Z, et al. How to measure and interpret quality improvement data. Clin J Am Soc Nephrol. 2016;11:908-914.

16. Siddiqi FS. Quality improvement in diabetes care: time for us to step up? Can J Diabetes. 2019;43:233.

17. Taylor MJ, McNicholas C, Nicolay C, et al. Systematic review of the application of the plan-do-study-act method to improve quality in healthcare. BMJ Qual Saf. 2014;23:290-298.

18. Ferdinand KC, Nasser SA. African American COVID-19 mortality: a sentinel event. J Am Coll Cardiol. 2020;75:2746-2748..

19. Muniyappa R, Gubbi S. COVID-19 pandemic, coronaviruses, and diabetes mellitus. Am J Physiol Endocrinol Metab. 2020;318:E736-E741.

20. Ebekozien OA, Noor N, Gallagher MP, Alonso GT. Type 1 diabetes and COVID-19: preliminary findings from a multicenter surveillance study in the U.S. Diabetes Care. 2020;43:e83-e85.

21. Majidi S, Ebekozien $\mathrm{O}$, Noor $\mathrm{N}$, et al. Inequities in health outcomes among patients in the T1D Exchange-Ql Collaborative. Diabetes. 2020;69(Supplement 1):1220-P. https://doi.org/10.2337/ db20-1220.-P.

22. Williams DR, Mohammed SA. Discrimination and racial disparities in health: evidence and needed research. I Behav Med. 2009;32:20-47.

23. Centers for Medicare \& Medicaid Services. Accountable Health Communities Model. CMS.gov web site. https://innovation.cms. gov/innovation-models/ahcm. Accessed October 10, 2020. 